\title{
L'impact de l'usage du TBI sur la motivation et les résultats scolaires des élèves dans l'enseignement des mathématiques et des sciences à l'école élémentaire
}

\section{The impact of the use of the IWB on students' motivation and academic performance in teaching mathematics and science in elementary school}

\author{
Abdessamad Redouani ${ }^{1 *}$ \\ ${ }^{1}$ Equipe de recherche "Tec\& Co" LISEC - EA 2310, INSPÉ de l'Académie de Strasbourg. France.
}

\begin{abstract}
Résumé. L'objectif de cette recherche est de mesurer l'impact de l'usage du TBI sur la motivation et les résultats scolaires des élèves dans le cadre de l'enseignement des mathématiques et des sciences à l'école élémentaire. Ce sont 121 élèves et 7 enseignants qui ont été interrogés et/ou observés pour cette recherche. Quatre instruments de collecte de données ont été utilisés : Un questionnaire d'enquête auprès des élèves, des entretiens individuels auprès des enseignants, des entretiens de groupe auprès des élèves et des observations directes en classe. Les résultats montrent que l'usage du TBI renforce la motivation et améliore les résultats scolaires des élèves en mathématiques et en sciences.
\end{abstract}

\begin{abstract}
The objective of this research is to measure the impact of the use of the IWB on the motivation and academic performance of students in the teaching of mathematics and science in elementary school. 121 students and 7 teachers were interviewed and / or observed for this research. Four data collection instruments were used: A student survey questionnaire, individual interviews with teachers, group interviews with students and direct classroom observations. The results show that the use of the IWB strengthens motivation and improves students' academic performance in math and science.
\end{abstract}

\section{Introduction}

*Corresponding author: abdessamad.redouani@etu.unistra.fr 
Les salles de classes dans les établissements scolaires sont de plus en plus équipées de technologies dites « éducatives » telles que les ordinateurs, les tablettes tactiles..., mais aussi les tableaux blancs interactifs (TBI). Le TBI, appelé aussi TNI (Tableau Numérique Interactif) ou TPI (Tableau Pédagogique Interactif) ou simplement TI (Tableau Interactif), est un tableau sur lequel il est possible d'afficher l'écran d'un ordinateur et de contrôler certaines de ses fonctions, directement à l'aide d'un stylet ou avec le bout des doigts. Il s'agit d'un dispositif qui « allie les avantages d'un écran tactile et de la vidéoprojection » [1, p.33]. L'apparition du VPI (Vidéoprojecteur Interactif) permet d'offrir à l'enseignant les mêmes fonctions que celles du TBI avec la possibilité supplémentaire de projeter le contenu de son cours sur un tableau blanc ordinaire [2].

En France, le rapport établi par le député parlementaire Jean-Michel Fourgous considère la présence des TBI dans les établissements scolaires comme un indicateur primordial de modernisation de l'école. Ainsi, dans ses 70 mesures pour réussir l'école numérique, Fourgous a mis en deuxième place : "Généraliser à 100\% des établissements scolaires les tableaux numériques interactifs associés à un ordinateur et à un logiciel de création de séquences pédagogiques multimédias $»[3$, p. 10].

C'est dans le cadre de nos interventions dans des écoles élémentaires publiques en France que nous avons découvert de près cet outil. Nous avons ainsi décidé de faire de l'usage du TBI en éducation notre objet de recherche avec l'intention d'en évaluer les potentielles plusvalues pédagogiques. Étant donné que l'étude de l'impact d'un tel outil pédagogique sur l'enseignement et l'apprentissage en général pourrait apparaître trop diffus, nous avons décidé de cibler deux disciplines importantes à l'école élémentaire, à savoir les mathématiques et les sciences. Ces deux disciplines sont riches en concepts abstraits souvent difficiles à comprendre. L'usage du TBI est potentiellement susceptible de les rendre plus accessibles.

\section{2 État de l'art}

Nous avons fait une recherche sur les travaux qui ont été réalisés au sujet du TBI. Il s'agit d'un sujet qui a été très abordé durant la dernière décennie. Plusieurs chercheurs soulignent fortement les avantages du TBI. C'est un outil technologique connecté à l'internet qui permet une meilleure présentation de contenus théoriques de façon multi-sensorielle [4]. Il permet à l'enseignant, contrairement au tableau classique, de sauvegarder de contenu pour le récupérer ultérieurement et de le partager ou de l'archiver [5]. Des chercheurs [6,7] suggèrent que l'utilisation d'un TBI réduit le temps passé à préparer le matériel didactique. En outre, les enseignants peuvent facilement interagir et communiquer avec les élèves et les engager davantage dans la leçon en utilisant les fonctionnalités de TBI [8]. Les effets du TBI sur les élèves peuvent être classés selon trois catégories. Des « effets conatifs (intérêt, enthousiasme, curiosité), des effets cognitifs (maintien de l'attention) et des effets sur les interactions » [9, p.81). En effet, le TBI « permettrait d'activer des conflits sociocognitifs susceptibles de favoriser l'apprentissage » [10, cité par 1, p. 33). L'utilisation des TBI peut accroitre la motivation des élèves au moins à court terme [11]. Le TBI aide les enseignants à attirer l'attention des apprenants [12]. Néanmoins, l'attention accrues des élèves avec le TBI ne signifie pas nécessairement que leur attention est orientée vers le contenu des apprentissages [13].

Bien que ces chercheurs montrent que le TBI présente un réel potentiel pédagogique, d'importants problèmes liés à son utilisation ont été signalés. La surutilisation du TBI en tant qu'outil de présentation dans un cours présenté de manière magistrale par l'enseignant peut détériorer la motivation et l'attention des élèves [14]. D'ailleurs, des chercheurs [15] ont démontré qu'il y a parfois un certain conflit entre les pédagogies actives centrées sur l'apprenant et l'usage du TBI où l'enseignant est le plus souvent devant la classe en train de 
présenter le cours de façon magistrale [16]. Les problèmes techniques et la rareté des ressources disponibles sont aussi classés parmi les défis majeurs relatifs à l'usage de TBI en salle de classe. Ainsi, des chercheurs stipulent que les enseignants passent plus de temps à gérer les problèmes techniques inhérents à l'usage de TBI qu'à se préoccuper de ce que les élèves apprennent [17]. De plus, l'usage d'un tel outil est considéré comme chronophage pour les enseignants dont les responsabilités professionnelles sont déjà importantes [18]. De même, l'étude menée par Pacurar a montré que l'utilisation des fonctionnalités de TBI dépend des disciplines d'enseignement [19]. Ainsi, selon cette étude, les enseignants d'histoire-géographie et ceux des langues n'utilisent que les fonctionnalités simples de TBI, alors que les enseignants des sciences et des mathématiques font une utilisation plus avancées et plus complexe des affordances du TBI.

\section{Cadre théorique}

\subsection{La motivation}

La définition admise de la motivation consiste à la décrire comme étant « le construit hypothétique utilisé afin de décrire les forces internes et/ou externes produisant le déclenchement, la direction, l'intensité et la persistance du comportement » [20, p.18).

Nous disposons aujourd'hui d'une multitude de théories sur ce thème. Ainsi, on distingue entre les théories des besoins (s'intéressant à la satisfaction des besoins), les théories du choix cognitif (s'intéressant à la perception des liens entre efforts, performances et récompenses), les théories des buts (les buts ont un rôle motivationnel), les théories de l'équité (équilibre entre le travail fourni et la récompense perçue) et les théories du renforcement béhavioriste (schéma réponse /récompense) [21].

En contexte scolaire, et notamment dans le cadre des apprentissages, nous nous intéressons plus particulièrement à comprendre pourquoi les élèves perdent-ils progressivement le goût et le plaisir d'apprendre au fur et à mesure de leur cheminement scolaire ? Ainsi les psychologues font la distinction entre trois types de motivation : la motivation intrinsèque, la motivation extrinsèque et l'amotivation. " La motivation intrinsèque renvoie à la pratique volontaire d'une activité pour l'intérêt qu'elle présente en elle-même et en l'absence de récompense extérieure » [22, cité par 23, p.9]. Elle consiste ainsi à faire une activité ou réaliser une tâche pour le plaisir inhérent à celle-ci. " La motivation extrinsèque se réfère à l'engagement dans un but non inhérent à l'activité soit en vue de retirer quelque chose de plaisant, soit afin d'éviter quelque chose de déplaisant » [24, cité par 23, p.9]. Cela veut dire que la réalisation d'une tâche dépend de l'atteinte d'un objectif détaché de l'action. L'amotivation représente l'absence de la motivation. Elle correspond à un état d'absence d'intention d'émettre un comportement. L'individu ne perçoit donc pas de raison d'agir [25].

Pour Bandura la perception que les personnes ont de leur efficacité est le fondement de leur motivation. C'est donc « l'expectation du résultat et le sentiment d'auto-efficacité d'un individu qui affectent la quantité d'efforts dépensés en vue de s'engager dans une activité quelconque » [26, p. 52]. 


\subsection{L'interactivité}

Comme son nom l'indique, le TBI est un outil pédagogique interactif. L'interactivité est définie comme une activité de dialogue entre un être humain et un programme informatique [27]. Les auteurs distinguent entre interactivité fonctionnelle et interactivité intentionnelle [28]. La première concerne l'échange d'information entre l'utilisateur et la machine, et la deuxième est liée à l'engagement de l'auteur face à l'utilisateur. Geneviève Jacquinot parle d'interactivité transitive qu'il oppose à l'interactivité intransitive [29]. La première est celle qui gère le protocole de communication entre l'utilisateur et la machine et la deuxième est celle qui gère le protocole de communication entre l'auteur et l'utilisateur. L'auteur absent mais présent à travers le logiciel qu'il a conçu [30].

L'interactivité est donc l'un des principaux avantages demandés au TBI [17]. Ainsi, cet outil augmente à la fois l'interaction enseignant-élèves, et élèves-élèves [31]. Le TBI favorise l'interaction enseignant-élèves grâce aux différentes façons de présenter les informations par l'enseignant [17]. L'interaction élèves-élèves est plus élevée chez les élèves qui apprennent en utilisant le TBI que chez ceux qui apprennent en suivant les méthodes classiques [32].

L'interactivité soutenue par le TBI a deux dimensions : interactivité technique et interactivité pédagogique [33]. Le TBI favorise le croisement entre ces deux types d'interactivité. L'interactivité technique assure l'interactivité pédagogique, quand par exemple, des images ou des films projetés obligent les élèves à discuter et à poser des questions [34].

\section{Problématique et hypothèses de recherche}

Dans cette étude nous cherchons à répondre à deux questions principales. La première porte sur l'impact de l'usage du TBI sur la motivation des élèves des écoles élémentaires dans le cadre de l'enseignement des mathématiques et des sciences, et la deuxième sur l'influence de cette usage sur leurs résultats scolaires.

En effet, les recherches antérieures stipulent que le TBI favorise la motivation des élèves à court terme et que cette motivation décroit à force d'utiliser cet outil [35]. Ces mêmes recherches suggèrent de poursuivre les études pour vérifier ces constats.

D'un autre côté, la plupart de ces recherches n'ont pas prouvé qu'il puisse y avoir un impact positif sur la réussite scolaire des élèves [16]. Pourtant, l'intégration pédagogique d'un tel outil n'aurait d'intérêt que dans la mesure où ses fonctionnalités sont exploitées dans le but d'améliorer les conditions de l'enseignement et celles de l'apprentissage.

De ces différentes considérations posons les hypothèses de recherche suivantes :

1. L'usage du TBI en classe augmente la motivation des élèves dans l'enseignement des mathématiques et des sciences à l'école élémentaire.

2. L'usage du TBI en classe a un impact significatif sur les résultats scolaires des élèves en mathématiques et en sciences à l'école élémentaire. 


\section{Méthodologie}

Pour vérifier nos hypothèses, notre étude repose sur une méthode de recherche dite mixte (mixed-method). Elle consiste à « mélanger » les deux méthodes : qualitative et quantitative. Le principe de base de cette méthodologie est que l'association des deux méthodes permet une meilleure utilisation des données. Ainsi, Postic revient sur la nécessité de ne plus opposer les méthodes qualitatives aux méthodes quantitatives [36]. Il considère que « la recherche quantitative et la recherche qualitative sont les deux versants d'un même toit » [37, p.114].

Le terrain de l'étude est composé de 3 écoles élémentaires publiques de Strasbourg en France. Ces écoles étaient équipées des TBI depuis la rentrée scolaire 2016/2017.

Pour réaliser notre enquête nous avons utilisé quatre outils de collecte de données, à savoir : un questionnaire quantitatif auprès des élèves (121 élèves), des entretiens de groupes « focus groups » (4 groupes de 4 élèves), des entretiens semi directifs individuels auprès des enseignants ( 7 enseignants) et des observations directes en classe ( 3 observations).

Durant ces observations, nous avons suivi des enseignants avec leurs élèves en utilisant le TBI dans deux séances de mathématiques et une séance de sciences.

La première séance de maths portait sur les fractions pour les élèves de CM1 (âgés de 10 et 11 ans). Durant cette séance l'enseignante a utilisé le TBI pour présenter cette notion compliquée pour les élèves de cet âge.

La deuxième séance observée portait sur les types et les caractéristiques des triangles pour les élèves de CM1/CM2 (classe combinée). Durant cette séance, l'enseignant n'a utilisé que le TBI pour réaliser l'exercice des triangles, malgré l'existence d'un tableau à craie dans la salle.

En ce qui concerne la séance observée en sciences, elle portait sur le système solaire pour les élèves de CM1. Pendant cette séance, l'enseignante a exposé plusieurs images des planètes sur le TBI en faisant passer les apprenants pour les nommer.

\section{Principaux résultats}

\subsection{L'impact du TBI sur la motivation des élèves en mathématiques et en sciences}

\subsubsection{La perception par les enseignants de l'impact du TBI sur la motivation des élèves dans l'apprentissage des mathématiques et des sciences}

En répondant à la question : «Quel est selon vous l'impact du TBI sur la motivation des élèves dans l'apprentissage des mathématiques et des sciences? », les enseignants ont déclaré que le fait d'utiliser le TBI en classe rend les élèves plus contents en les incitants à aller au tableau. De plus, le TBI leur donne plus d'envie de participer aux cours. D'autre part, le TBI augmente l'intérêt que portent les élèves au cours : « Moi je pense que les enfants sont plus intéressés en utilisant la nouvelle technologie » (Enseignante2). De plus, des enseignants ont dit que le fait de venir au TBI est motivant pour les élèves, d'autre stipulent que les élèves voient le TBI comme un outil ludique ce qui fait que cela leur donne plus de motivation. De son côté, une enseignante a avancé que le TBI facilite l'apprentissage des élèves ayant des difficultés : "Par exemple, ceux qui ont du mal à écrire. Ils appuient sur un bouton, ils déplacent une flèche, même pour eux la communication est plus facile ». (Enseignante 5). Le TBI aide aussi selon les enseignants à capter l'attention des élèves : " C'est sûr que pour 
capter l'attention des élèves un TBI de nos jours c'est plus facile qu'un tableau vert ». (Enseignante 5).

Les observations directes en classe, que nous avons réalisées, nous ont permis de confirmer les propos des enseignants concernant la motivation des élèves. Nous avons ainsi constaté une forte demande des apprenant pour passer au TBI.

\subsubsection{La perception par les élèves de l'impact du TBI sur leur motivation}

Par le biais du questionnaire, nous avons posé aux élèves une série de questions concernant l'impact du TBI sur leur motivation. Les graphiques suivants représentent les résultats obtenus pour certaines de ces questions :

Q : Lorsque le cours est fait avec TBI, tu as envie d'aller au tableau plus que lorsque le cours est fait avec un tableau classique?

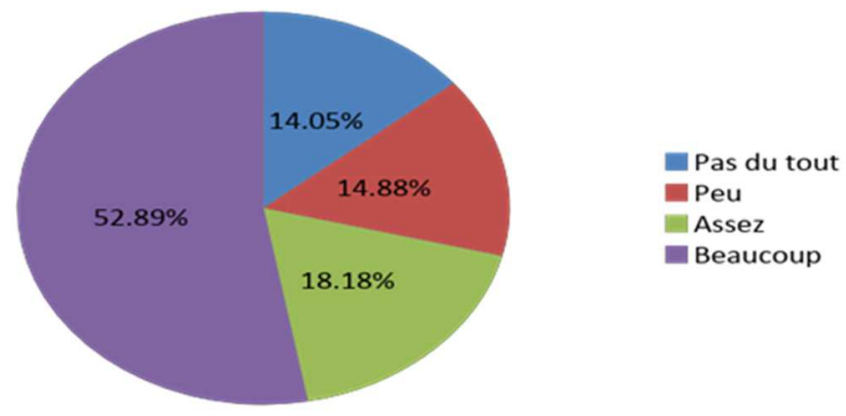

Fig. 1 La perception par les élèves de l'impact du TBI sur leur envie d'aller au tableau

Q : Lorsque la maîtresse (le maitre) utilise le TBI, ton envie de suivre le cours augmente ?

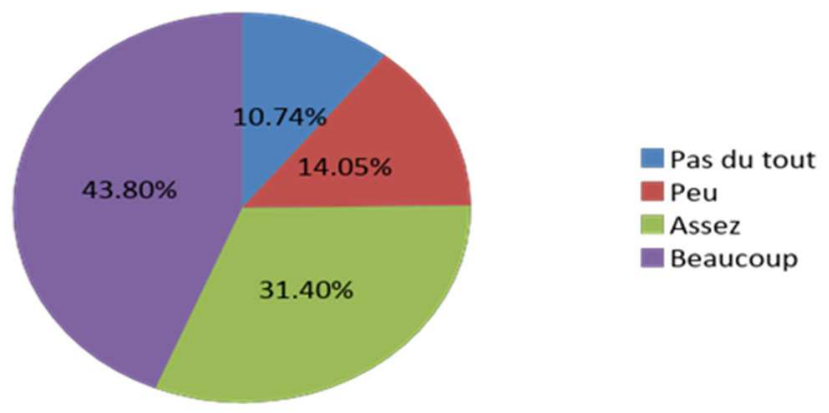

Fig. 2 La perception par les élèves de l'impact du TBI sur leur envie de suivre le cours

Pour mieux expliquer ces résultats, nous avons demandé aux élèves, durant les entretiens en groupes, comment le TBI influence-t-il leur envie d'apprendre. La plupart des élèves ont indiqué qu'ils sont très motivés pour apprendre avec le TBI. Les raisons de cette motivation augmentée diffèrent d'un élève à l'autre.

En effet, le principal facteur qui attire les élèves au TBI reste le caractère numérique de l'outil. Aussi, le fait que le TBI permet de découvrir beaucoup de choses et d'apprendre avec des supports multi-sensoriels donne plus d'envie aux élèves. 


\subsection{L'impact du TBI sur les résultats scolaires des élèves en mathématiques et en sciences}

\subsubsection{La perception par les enseignants de l'impact du TBI sur les résultats scolaires des élèves en mathématiques et en sciences}

Nous avons demandé aux enseignants quel était l'impact de l'utilisation du TBI en classe sur les résultats scolaires des élèves en mathématiques et en sciences. Les 7 enseignants interviewés ont été incapables de dire si le TBI a un impact significatif sur les résultats scolaires des apprenants. Cependant, ils ne pensent pas que cela puisse avoir un effet négatif. De plus, une enseignante a considéré que si la concentration et la motivation sont meilleures, et si les élèves sont plus actifs alors ils auront de meilleurs résultats. D'autres enseignants pensent que puisque l'on gagne du temps grâce au TBI cela peut aider à avoir de meilleurs résultats, surtout pour les élèves en difficulté, en leur consacrant plus du temps.

D'autres enseignants ne perçoivent pas le TBI comme un vecteur qui améliore les résultats scolaires des élèves.

\subsubsection{La perception par les élèves de l'impact du TBI sur leurs résultats scolaires en mathématiques et en sciences}

Nous avons demandé aux élèves à quelle fréquence leurs résultats scolaires ont été améliorés par l'utilisation du TBI en mathématiques puis en sciences. Les graphiques suivants représentent les résultats obtenus pour chacune des questions :

Q. Après l'arrivée de TBI dans ta classe tes résultats en Maths ont-ils été améliorés ?

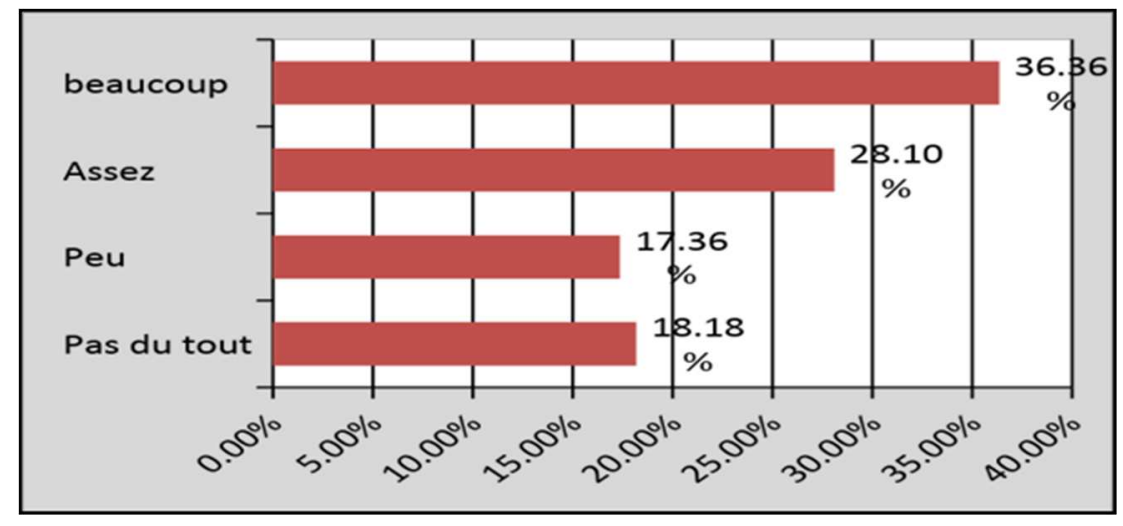

Fig. 3 La perception par les élèves de l'impact du TBI sur leurs résultats en mathématiques

Q. Après l'arrivée de TBI dans ta classe tes résultats en sciences ont-ils été améliorés ? 
Fig. 4 La perception par les élèves de l'impact du TBI sur leurs résultats en sciences

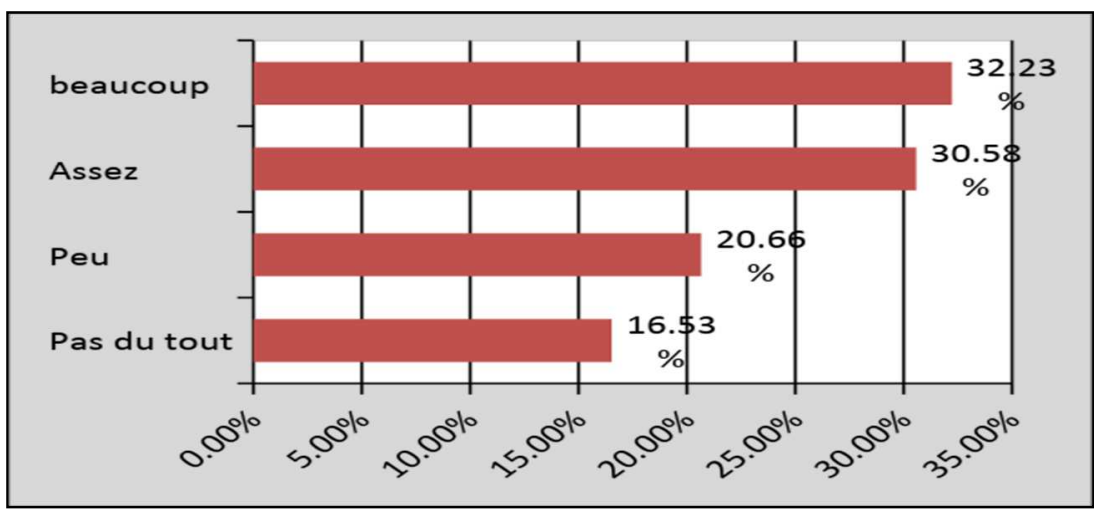

En analysant les résultats obtenus par ces questions, nous constatons que les pourcentages des élèves qui perçoivent que le TBI améliore beaucoup ou assez leurs résultats scolaires, dans les deux matières, sont importants.

Cependant, lors des entretiens de groupes les réponses des élèves par rapport à ce point étaient différentes. Ainsi, en répondant à la question : Quelle est l'influence de TBI sur tes résultats scolaires en mathématiques et en sciences ? Les élèves ont donné des réponses différentes. Certains élèves pensent que le TBI influence positivement leurs résultats scolaires. D'autres élèves pensent que le fait d'avoir un TBI dans la classe ne change pas grands choses dans leurs résultats : "Ça ne change rien. [...] Si tu as envie de travailler que ce soit avec le TBI ou le tableau à craie tu auras des bonnes notes » (élève). Il est à signaler qu'aucun élève n'a évoqué un effet négatif du TBI sur ses résultats scolaires dans les deux matières.

\section{Conclusion et perspectives}

Cette étude nous a permis de faire le point sur l'impact de l'usage du TBI sur la motivation et les résultats scolaires des élèves à l'école élémentaire notamment dans le cadre de l'enseignement des mathématiques et des sciences.

Les résultats de notre enquête montrent que le TBI constitue un facteur donnant envie aux élèves de participer au cours et d'apprendre grâce à son caractère numérique mais aussi par le fait qu'il permet la découverte des choses et l'apprentissage avec des supports multisensoriels. De plus, les élèves perçoivent que l'usage du TBI améliore leurs résultats scolaires dans les deux disciplines visées par notre étude.

Cependant, le fait que les enseignants à l'école élémentaire sont polyvalents ne nous a pas permis d'avoir des réponses spécifiques aux deux matières visées. Cela peut constituer une limite à notre recherche.

Dans le cadre des perspectives de ce travail, nous envisageons de faire une recherche sur l'impact de l'usage du TBI sur l'attention des élèves. C'est l'un des éléments les plus importants dans le processus de l'apprentissage. Il s'agit d'une des capacités les plus attendues par les enseignants et les formateurs. Ainsi, le débat sur l'impact de l'usage des appareils numériques sur l'attention des apprenants dans les salles de classe est un sujet d'actualité. De plus, la question de l'attention des élèves suscite un vif intérêt, à l'ère du numérique, aussi bien chez les chercheurs que chez les praticiens. 


\section{Références}

1. M. Trestini. La modélisation d'environnements numériques d'apprentissage de nouvelle génération. Londres : ISTE Editions (2018)

2. A. Redouani. L'effet de l'usage du Tableau Blanc Interactif (TBI) sur les pratiques pédagogiques dans trois écoles élémentaires de Strasbourg. Médiations et Médiatisations. Revue internationale sur le numérique en éducation et communication, 5. https://revue-mediations.teluq.ca/index.php/Distances/article/view/168. (2021)

3. J.M. Fourgous. Réussir l'école numérique - Rapport de la mission parlementaire sur la modernisation de l'école par le numérique. Paris : Mission parlementaire de Jean-Michel Fourgous sur l'école numérique (2010).

4. F. Saltan, k. Arslan. A New Teacher Tool, Interactive White Boards: A Meta Analysis. In I. Gibson, R. Weber, K. McFerrin, R. Carlsen, D. Willis (Eds.), Proceedings of SITE 2009--Society for Information Technology \& Teacher Education International Conference (pp. 2115-2120). Charleston, SC, USA: Association for the Advancement of Computing in Education (AACE). Retrieved December 7, 2018 from https://www.learntechlib.org/primary/p/30936/. (2009)

5. J. Dostal. Reflections on the use of interactive whiteboards in instruction in international context. The New Educational Review, 25(3), 205-220, (2011)

6. P. Levy, Interactive whiteboards in learning and teaching in two Sheffield schools: A developmental study, (2002)

7. A. Tozcu, The use of interactive whiteboardsin teaching non-roman scripts. Computer Assisted Language Learning, 21(2), 143-166. doi:10.1080/0958822080 1943726, (2008)

8. Becta. What the research says about interactive whiteboards. The British Educational Communications and Technology Agency, Coventry, England. (2003)

9. L. Boulc'H, G. L. Baron. Connaissances et représentations du Tableau Numérique Interactif chez les futurs professeurs des écoles : Réflexions sur la formation aux technologies éducatives. Sciences et technologies de l'information et de la communication en milieu éducatif: Analyse de pratiques et enjeux didactiques., , Patras, Grèce. pp.75-86. ffedutice-00676175f, (2011)

10. M. Gilly. Approche socio-constructive du développement cognitif de l'enfant d'âge scolaire, In D. Gaonac'h\& C. Golder, Manuel de Psychologie pour l'enseignement, Hachette, Paris, , (1995)

11. D. Glover, D. Miller, Running withTechnology : the pedagogic impact of the large-scale introduction of interactive whiteboards in one secondary school. Journal of Information Technology for Teacher Education (2001)

12. K. Wall, S. Higgins, H. Smith. « The visual helps me understand the complicated things »: Pupil views ofteaching and learningwith interactivewhiteboards. British Journal of Educational Technology, 36(5), 851-867. doi:10.1111/j.1467- 8535.2005.00508.x, (2005)

13. S. Higgins, G. Beauchamp, D. Miller. Reviewing the literature on interactive whiteboards. Learning. Media and Technology, 32(3),

213-225. doi:10.1080/17439880701511040, (2007)

14. I. Hall, S. Higgins, Primary school students' perceptions of interactive whiteboards. Journal of Computer Assisted learning, (2005)

15. M. N. Khambari, D, Hassett, M. Thomas, S.L. Wong. Interactive whiteboards in classrooms: Debates, issues, and impeding factors, (2014) 
16. T. Karsenti, Le tableau blanc interactif (TBI) : usages, avantages et défis. Montréal : CRIFPE, (2016)

17. S. Lefebvre, G. Samson, Le tableau numérique interactif: Quand chercheurs et praticiens s'unissent pour dégager des pistes d'action. Presses de l'université de Québec, (2015)

18. E. Manny-Ikan, O. Dagan, T.B. Tikochinski, R. Zorman, Using the interactive white board in teaching and learning - An evaluation of the SMART CLASSROOM pilot project. Interdisciplinary Journal of E-Learning and Learning Objects, 7, 249-273, (2011)

19. E. Pacurar, L'appropriation du tableau numérique interactif et du manuel numérique chez des enseignants du second degré : valeurs ressources et affordances contextuelles, revue La Recherche en éducation, AFIRSE, ISSN 16470117, nr. 13. (2015)

20. R.J. Vallerand, E.E. Thill, Introduction à la psychologie de la motivation. Laval : Éditions Études Vivantes (1993)

21. C. Levy-Leboyer, Les crises de la motivation. PUF (1984)

22. E. Deci, M. Ryan, Self-determination theory and the intrinsic motivation. Social development and well-being. American psychologist, (2000)

23. N. Leroy, G Joët, La motivation des élèves en difficulté. Des processus aux interventions. Séminaire de rentrée de la Mission Générale d'Insertion de l'Académie de Grenoble, Autrans, (2005)

24. E. Deci, M. Ryan, Intrinsic motivation and self-determination in human behavior, New York, Plenium, (1985)

25. R. J. Vallerand, An integrative analysis of intrinsic and extrinsic motivation in sport. Journal of applied sport psychology, (1999)

26. T. Karsenti, Étude de l'interaction entre les pratiques pédagogiques d'enseignants du primaire et la motivation de leurs élèves, Thèse de doctorat présentée à l'Université du Québec à Montréal, (1998)

27. G. Vidal, Usages de l'innovation interactive. Document numérique, vol. 10,(3), 11-23. https://www.cairn.info/revue-document-numerique-2007-3-page11.htm, (2007)

28. J.T. Julia, Interactivité, modes d'emploi. Réflexions préliminaires à la notion de document interactif. Documentaliste-Sciences de l'Information. (Vol. 40), p. 204-212, (2003)

29. G. Jacquinot, C. Meunier, Introduction. L'interactivité au service de l'apprentissage. Revue des sciences de l'éducation 251: 3-15. DOI : 10.7202/031990ar, (1999)

30. J.Y. Petitgirard, D. Abry, E. Brodin, Le tableau blanc interactif. NATHAN. Collection: Techniques et Pratiques de classe, (2011)

31. C. Lewin, B. Somekh, S. Steadman, Embedding interactive whiteboards in teaching and learning: The process of change in pedagogic practice. Education and Information Technologies, 13 (4), 291-303, (2008)

32. F. J. Zittle, Enhancing Native American Mathematics Learning: The Use of Smartboard generated Virtual Manipulatives for Conceptual Understanding, (2004)

33. H. J. Smith, S. Higgins, K. Wall, J. Miller, Interactive whiteboards: Boon or bandwagon? A critical review of the literature. Journal of Computer Assisted Learning, 21(2), 91101, (2005)

34. G. Beauchamp, Kennewell. Interactivity in the classroom and its impact on learning, Computers \& Education, 54(3), p.759-766, (2010) 
35. N. Balta, M. Duran, Attitudes of students and teachers towards the use of interactive whiteboards in elementary and secondary school classrooms. Turkish Online Journal of Educational Technology, 14(2), 15-23. R. Nicole, Title of paper with only first word capitalized, J. Name Stand. Abbrev., in press. (2015)

36. M. Postic, J.M. De Ketele, Observer les situations éducatives. In : Revue française de pédagogie. Volume 89. pp. 112-115, (1989)

37. M. Altet, M. Postic , J.M. De Ketele, Observer les situations éducatives . In: Revue française de pédagogie, volume 89, 1989. pp. 112-115 (1989) 